\title{
The effects of selective ibotenate lesions of the hippocampus on conditioned inhibition and extinction
}

\author{
K.-H. CHAN \\ Hartwick College, Oneonta, New York \\ L. E. JARRARD \\ Washington and Lee University, Lexington, Virginia \\ and \\ T. L. DAVIDSON \\ Purdue University, West Lafayette, Indiana
}

\begin{abstract}
Previously, Solomon (1977) reported that aspiration lesions of the dorsal hippocampus in rabbits had no effect either on the acquisition of Pavlovian conditioned inhibition or on performance during a subsequent retardation test. The present experiment confirmed and extended these findings by showing that rats with ibotenate lesions of the complete hippocampus (the dorsal and ventral hippocampus and the dentate gyrus) were also unimpaired on the same types of tasks. Additional tests with the same rats showed that removing the hippocampus significantly impaired extinction of responding to a stimulus that had been previously trained with an appetitive unconditioned stimulus. The performance of the lesioned rats on a summation test was also marginally, but not significantly, different from that of controls. The data are discussed with reference to the idea that the hippocampus is involved with the formation of some, but not all, types of inhibitory associations.
\end{abstract}

Solomon's (1977) report that aspiration lesions of the dorsal hippocampus had no effect on the acquisition of Pavlovian conditioned inhibition (CI) of the eye-blink response has had a strong influence on thinking about the role of the hippocampusin inhibitory learning. In Solomon's study, rabbits with hippocampal lesions and their controls were trained using a standard Pavlovian CI procedure in which the presentation of a tone conditioned stimulus (CS) signaled the occurrence of a shock unconditioned stimulus (US), except when the tone was presented in simultaneous compound with a light CS. Solomon reported that training on this $\mathrm{T}+$, $\mathrm{LT}-$ problem yielded no differences between lesioned and control animals in excitatory conditioning on $\mathrm{T}+$ trials or in the ability of the light to suppress conditioned responding on LT - trials. Furthermore, there was no difference between rats with hippocampal lesions and controls in the acquisition of conditioned responding when the rats were trained subsequently with the light as a signal for delivery of the US. If during original T+, LTtraining, more inhibitory conditioning accrued to the light for controls than for rats with a damaged hippocampus, this should have reduced or retarded subsequent excitatory conditioning of the light for controls, relative to rats with

Correspondence concerning this article should be addressed to K.-H. Chan, Department of Psychology, Hartwick College, 1 West Street, Oneonta, NY 13820 (e-mail: chank@hartwick.edu). hippocampal lesions. On the basis of these findings, Solomon concluded that an intact hippocampus is not required for the acquisition of Pavlovian CI. This conclusion contributed to the development of theories that moved away from the view that the hippocampus is an important substrate for inhibitory learning.

Although there is little reason to question Solomon's (1977) findings, a reexamination of the generality of his conclusions may be warranted on the basis of several considerations. First, in Solomon's study, only the dorsal hippocampi were removed, thus leaving open the possibility that the extent of hippocampal damage in his experiment was not large enough for impairments in hippocampaldependent inhibitory learning to be observed. Furthermore, the aspiration method used by Solomon is also known to cause damage to extra-hippocampal regions and to destroy axons that pass through the region of damage. Confidence in the generality of any conclusion about CI as a hippocampally independent or dependent process would be strengthened to the extent that it was based on assessment of the effects of hippocampal damage that was both more complete and more selective than the damage studied by Solomon.

Second, Solomon (1977) studied the effects of hippocampal lesions on inhibitory learning that emerged as a result of training within a Pavlovian T+, LT - conditioning procedure. There is now clear evidence that inhibitory learning also occurs with procedures that involve the for- 
mation of associative relations that are different from those that are formed on the basis of standard A+, ABtraining (see Williams, Overmier, \& LoLordo, 1992). More specifically, research has shown that a stimulus can enter into direct inhibitory association with a US (e.g., Rescorla, 1969) or into concurrent excitatory and inhibitory association with a US (e.g., Barnet \& Miller, 1996; Delameter, 1996; Matzel, Gladstein, \& Miller, 1988; Williams \& Overmier, 1988) or can modulate the activation of an inhibitory association between another CS and its US (e.g., Bouton, 1994; Holland, 1985). The generality of any conclusion about the effects of hippocampal damage on inhibitory learning is limited to the extent that it is based on only one of several conditions under which inhibitory learning is thought to occur.

Third, Solomon (1977) studied the acquisition by rabbits of conditioned eyeblink responding that was based on training with an aversive US. Hippocampal involvement (or lack thereof) in CI or any other general learning process can be confirmed only to the extent that similar patterns of findings are obtained with other species, response measures, and USs. The purpose of the present experiment was to reexamine the effects of hippocampal damage on the acquisition of Pavlovian CI. One goal of our study was to assess the generality of Solomon's finding that aspiration lesions of the dorsal hippocampus had no effect on CI of the rabbit eyeblink response on the basis of a shock US. To pursue this goal, instead of aspiration lesions of the dorsal hippocampus in rabbits, we used a neurotoxic lesion technique with rats that was not available at the time of Solomon's earlier study. This technique involves the intracranial infusion of minute quantities of ibotenic acid to permit relatively complete removal of all dorsal and ventral hippocampal cell fields (CA1 - CA3 plus the dendate gyrus) without producing significant collateral damage to extra-hippocampal areas or to fibers of passage (Jarrard, 1989). In addition to changing the species and the lesion procedure, we employed a sucrose pellet US and measured the performance of appetitive conditioned responses (CRs; i.e., food cup approach) instead of measuring eyeblink responses to index learning about a shock US.

Furthermore, like Solomon (1977), we assessed the effects of these more selective lesions on acquisition of inhibitory $\mathrm{T}+$, LT - conditioning and on performance during a subsequent $\mathrm{L}+$ retardation test. However, we also employed two additional tests to assess differences in inhibitory learning between ibotenate-lesioned and control rats. After the completion of T,$+ \mathrm{LT}-$ training, we trained white noise as a CS for the sucrose pellet US (N+ training). We then presented this cue in simultaneous compound with the light that had been trained as a conditioned inhibitor during initial $\mathrm{T}+, \mathrm{LT}-$ training. This procedure is called a summation test, because the performance of conditioned responses on $\mathrm{LN}$ - test trials is thought to reflect the summation of opposing inhibitory conditioning to the light and excitatory conditioning to the noise. Assuming that both lesioned and control rats show similar excitatory conditioning to the white noise, differences be- tween these groups in responding during the $\mathrm{LN}-$ test trials would indicate the existence of prior differences in inhibitory learning about the light.

After the conclusion of the summation test, extinction of responding to the noise CS was tested. Recent theories suggest that such a trained and extinguished CS is embedded in both an excitatory and an inhibitory association with the representation of the US (e.g., Bouton \& Nelson, 1998; Pearce, 1987; Wagner, 1981; see also Konorski, 1948). In contrast, the T+, LT - training procedure is thought to embed the light within an inhibitory association with the US, but because the US is never presented when light occurs, this type of training should not result in the formation of any strong excitatory light $\rightarrow$ US association. In a recent paper, Chan, Morell, Jarrard, and Davidson (2001) reviewed the results of a variety of studies that were consistent with the hypothesis that inhibitory learning about a CS that is also embedded in a concurrent excitatory association with the US is especially sensitive to hippocampal damage. To reduce the effect of differences in subjects and lesions, the present experiment was performed to assess, within the same rats, the effects of ibotenate lesions of the hippocampus on inhibitory learning that develops as a consequence of training with Pavlovian CI and extinction procedures, respectively.

Chan et al. (2001) proposed that the hippocampus may be involved with the formation of inhibitory associations involving cues that are already embedded in an excitatory association with the US. The $\mathrm{A}+, \mathrm{AX}$ - training procedure used by Solomon (1977) is designed to endow Stimulus X with inhibitory power. The actual US is not presented when $\mathrm{X}$ occurs, thus reducing the opportunity for direct excitatory association to develop between $X$ and the US. It may be that any "excitation" shown by X depends not on its direct association with the US representation, but on its association with other events, such as the A stimulus or the CR. On the other hand, the extinction procedure involves the formation of inhibitory associations to a cue that is already embedded in a direct excitatory association with the US. On the basis of the hypothesis put forth by Chan et al. we make the prediction that the same animals that show intact performance in the $\mathrm{A}+, \mathrm{AX}-$ procedure might be impaired in the extinction test.

\section{METHOD}

\section{Subjects}

The subjects were 16 naive male Sprague-Dawley rats obtained from Harlan Sprague Dawley, Inc., Indianapolis, IN. The rats were approximately 180 days old and weighed between 270 and 300 grams upon arrival to the laboratory. The subjects were housed individually in stainless steel cages and were maintained on a 12:12-h light:dark cycle with lights on at $0700 \mathrm{~h}$. The experimentation was carried out on every weekday, starting at about $1330 \mathrm{~h}$. Water was available ad lib in the home cage, and the subjects were maintained on Purina rat chow at $85 \%$ of their free-feeding body weights.

\section{Apparatus}

The four identical conditioning chambers used in this experiment were constructed of aluminum end walls and clear Plexiglas sides, measuring $21.6 \times 21.6 \times 27.9 \mathrm{~cm}$ (the animals were run in training 
and test session in squads of 4 animals). The floor of each chamber consisted of stainless steel bars, $0.48 \mathrm{~cm}$ in diameter, spaced $1.9 \mathrm{~cm}$ apart. A food cup was located in one end wall of each chamber. Each chamber was seated within a larger sound-attenuating enclosure. The tone conditioned stimulus was produced by a Radio Shack 2800-hz Piezo Alerting Buzzer (Cat. No. 273-068) located outside of the chamber by the end wall with the food cup. The visual CS was produced by a $6-\mathrm{W}$ jeweled panel light located $5 \mathrm{~cm}$ above and $2.5 \mathrm{~cm}$ to the left of the magazine in the conditioning chambers. The noise CS was an 80-db zero-frequency white noise generated by the ANL 926 module supplied by Med Associates.

\section{Data Collection}

Changes in behavioral activity were monitored with a computercontrolled infrared monitoring system. One pair of electronic photobeams (ENV-256C infrared photobeam controller and DIG-712 photobeam inputs, Med Associates, Inc.) lined each cage on the side walls in front of the magazine and approximately $2 \mathrm{~cm}$ above grid floor. These beams were controlled and the data analyzed with software developed in the lab for the measurement of appetitive activity. Approximately 30 times per second during the pre-CS and CS periods, the computer recorded binary values for photobeam interruptions in each box. The percentage of the time that the photobeam was interrupted during pre-CS and CS periods was recorded as mean appetitive behavior. The computer and interface operating the photobeams were located in an adjoining room.

\section{Procedure}

Surgical and histological procedures. Eight rats underwent the ibotenate lesioning procedure designed to remove selectively the cells that make up the hippocampus. A total of 32 injections were made at 16 different sites on each side of the hippocampus. A micropipette with a very fine tip was attached to the needle end of the microsyringe to reduce cortical damage resulting from the injections. The amount of IBO injected at each site varied from 0.05 to $0.12 \mu \mathrm{L}$. After each injection, the pipette was left in place for approximately $30-60 \mathrm{sec}$ to reduce spread of toxin up the tract. After injection at each site, the pipette was cleaned with saline on the outside, and a small amount of IBO was ejected through the tip to make sure the toxin had not dried up and the tip was not clogged. After all the injections had been made, the skull was cleaned and sutured back up, and the rat was allowed to recover on a heat blanket. Four rats underwent sham control operations and served as operated controls. In these rats, the glass pipette was lowered through the cortex at the same stereotaxic coordinates as those used for rats receiving ibotenate lesions of the hippocampus, but no IBO was delivered. The remaining rats were unoperated controls. The procedures used for lesioning the hippocampus have been described in detail elsewhere (Jarrard, 1989).

After completion of the experiment, all the lesioned animals were deeply anesthetized and perfused with physiological saline and formalin. The brains were removed from the skull and embedded in egg yolk, and some brains were cut in horizontal and some in coronal sections on a cryostat into $40-\mu \mathrm{m}$ sections. Every fifth section was stained with a cresyl-violet stain to determine cell loss due to the lesions.

Conditioning procedures. In the first phase of the experiment, all the subjects were given CI training in which a tone was always followed by US presentations (two sucrose pellets) and a simultaneous light-tone compound was not followed by any US presentation. Each session lasted $32 \mathrm{~min}$, and the subjects received two reinforced trials and six nonreinforced trials. The mean intertrial interval (ITI) was $240 \mathrm{sec}$, with a range of $120-360 \mathrm{sec}$. In the next phase, the subjects were given six training sessions with a noise CS, followed by US presentations. The animals received eight trials per session. The session length and the ITI were the same as those in the first phase. In the next phase, the subjects were given four trials of noise presentations and four trials of a summation test in which the inhibitor (light) was presented in compound with the transfer target (noise), and no US was presented in this phase. The trial sequence employed in the summation test was ABBAABBA, where A and B (i.e., noise alone and light-noise compound) were counterbalanced. After the summation test, all the subjects were given six sessions of nonreinforced presentations of the noise CS. There were eight trials in each session with the same session length and mean ITI as those in the previous phases. After the extinction phase, all the subjects received four sessions of the original CI training before they were given a retardation test. In the eight sessions of the retardation test phase, all the subjects received eight trials of paired presentations of the inhibitor (light) and the US.

\section{RESULTS}

\section{Surgical and Histological Results}

Inspection and evaluation of the cell loss resulting from the surgical procedures described above indicated that all animals in the hippocampal lesion group had extensive loss of cells in the hippocampus (CA1-CA3 pyramidal cells, dentate granule cells, and interneurons). The cell loss within the hippocampus was complete in the lesioned animals and was limited to the structure. Although there were 3 animals that had some minor sparing of pyramidal cells and/or dentate granule cells, these cells were few in number and were confined mostly to the posterior-ventral hippocampus. There was minor loss of cells in the adjacent subicular complex in 3 animals, but cell loss to this area was limited and unilateral. The damage described above is essentially similar to that found in other experiments in which the same surgical procedure was used (e.g., Benoit, Davidson, Chan, Trigilio, \& Jarrard, 1999; Jarrard, 1989). The animals in the operated control group had no noticeable damage resulting from lowering the glass pipette through the cortex (see Figure 1).

\section{Behavioral Results}

Conditioned inhibition training. The responding to the CSs from the acquisition phase is shown in Figure 2. There were no main effects or interactions involving type of control (sham vs. unoperated) as a factor (largest $F=$ 5.19 , for surgery $\times$ period interaction during the conditioned inhibition phase). Therefore, the data from the sham controls and the unoperated controls were collapsed together for the remaining analyses. The 3 rats with minor sparing of the ventral posterior hippocampus were compared against the remaining lesioned rats, and there was no significant main effect or interaction involving lesion extent as a factor (largest $F=3.31$, for the trial block $\times$ lesion extent interaction during the conditioned inhibition phase). The lesioned and the control rats did not differ in their levels of responding during the intertrial intervals (largest $F=1.406$, for the surgery $\times$ trial block interaction during the extinction phase). As a result of 20 training sessions, both the hippocampal-lesioned and the control rats learned to make conditioned responses to the tone and refrained from responding when the light-tone compound was presented. Multiple analyses of variance (ANOVAs) involving surgery as a between-subjects factor and trial type (A+ vs. XA-), session (1-20), and trial 

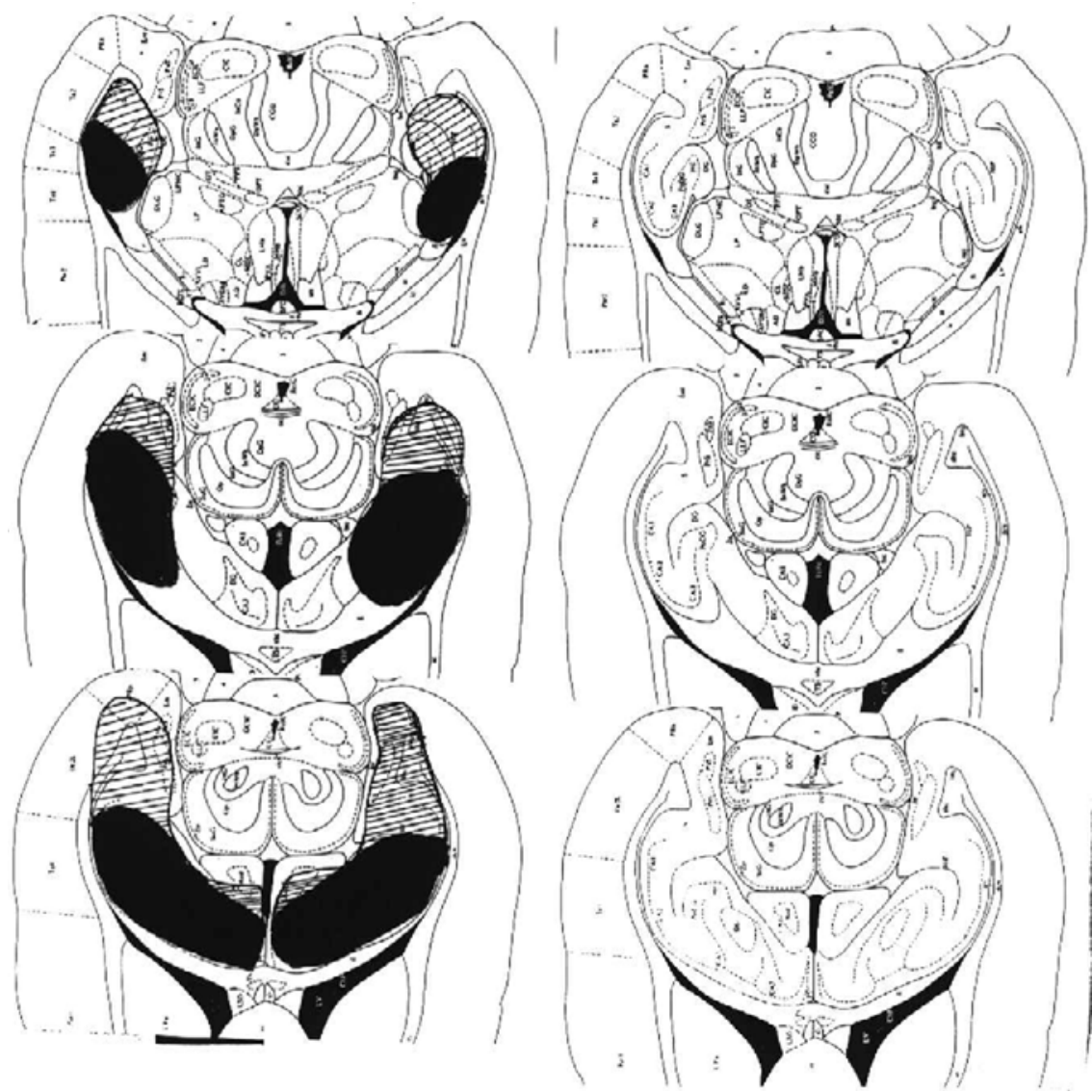

Figure 1. Serial horizontal sections illustrating the extent of maximal (black) and minimal (gray) lesions of the hippocampus (left), as compared with intact controls (right). Sections are arranged from ventral (top) to dorsal (bottom). From The Rat Brain in Stereotaxic Coordinates, by G. Paxinos and C. Watson, 1998, San Diego: Academic Press. Copyright 1998 by Academic Press. Adapted with permission.

block (1 or 2) as within-subjects factors confirmed these observations. The statistical analyses revealed significant main effects of trial type $[F(1,14)=301.5, p<.01]$ and session $[F(9,126)=9.6, p<.01]$. There was also a significant interaction involving trial type and session $[F(9,126)=11.7, p<.01]$. There was no significant main effect or interaction involving surgery (largest $F=1.15$, for the surgery $\times$ trial type interaction).

Transfer target training. Both the lesioned and the control rats increased the levels of conditioned responding from Day 1 to Day 6 of training. The lesioned and the control rats did not differ during the course of the training of the transfer target, except that the lesioned rats showed slightly more responding than did the controls during the pre-CS period. Multiple ANOVAS using surgery (Hip vs. Con) as a between-subjects factor and block (1-12), trial (1-4), and period (pre-CS vs. CS) as within-subjects factors confirmed these observations. There were significant main effects of trial $[F(3,42)=3.02, p<.05]$ and period $[F(1,14)=106.55, p<.01]$ and significantblock $\times$ period $[F(11,154)=2.23, p<.05]$, surgery $\times$ block $\times$ period $[F(11,154)=2.09, p<.05]$, and surgery $\times$ trial $\times$ period
$[F(3,42)=3.09, p<.05]$ interactions. There was no other significant main effect or interaction involving surgery and period as factors (largest $F=2.5$, for the surgery main effect). Separate analysis of pre-CS versus CS periods revealed a significant surgery $\times$ block $\times$ trial interaction $[F(33,462)=1.55, p<.05]$ during the pre-CS period but no significant main effect or interaction involving surgery during the CS period.

Summation test. The transfer tests involved presenting the noise either alone or in simultaneous compound with the inhibitor (light). In the summation test, the stimuli were presented without reinforcement. As a result, the subjects also had the opportunity to learn extinction to the stimuli. We analyzed the data from the first two trials of each session. This made it easier to compare our results with those of Holland, Lamoureux, Han, and Gallagher (1999), which also analyzed two trials of the summation test session.

Figure 3 shows the mean conditioned responses during the first two trials of the summation test by the control and the lesioned rats to the noise alone and to the light-noise compound. The figure reveals that both the hippocampallesioned and the control rats responded more to the noise 


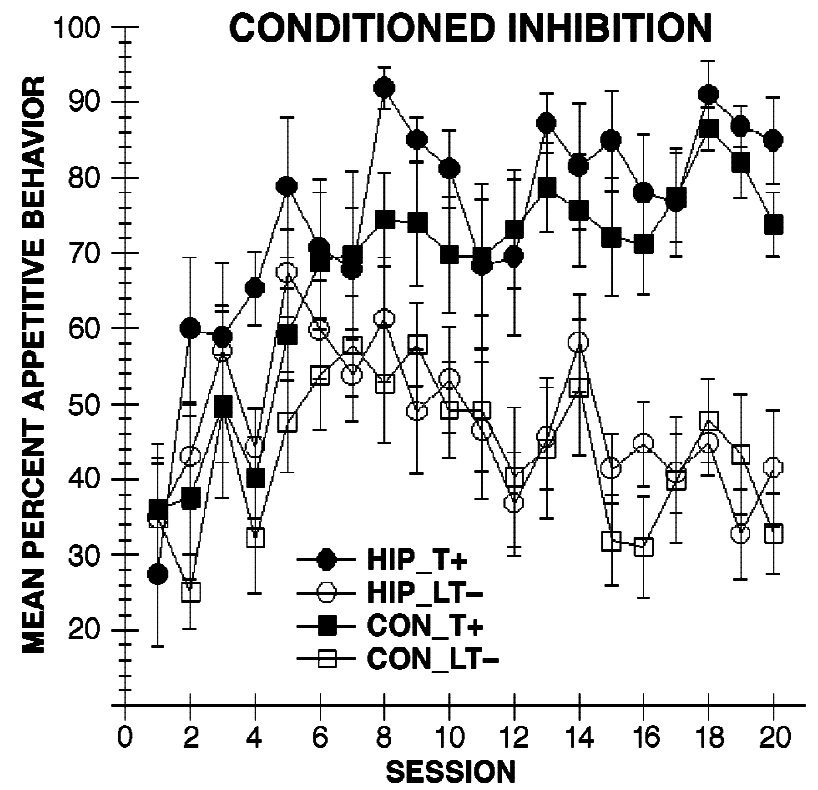

Figure 2. Acquisition of appetitive behavior by hippocampallesioned and control rats in a conditioned inhibition (CI) task (i.e., $\mathrm{T}+, \mathbf{L T}-$ ). The closed circles represent appetitive behavior by lesioned rats during $T+$ trials. The open circles represent appetitive behavior by lesioned rats during LT - trials. The closed squares represent appetitive behavior by control rats during $\mathrm{T}+$ trials. The open squares represent appetitive behavior by control rats during $\mathbf{L T}-$ trials.

alone than to the light-noise compound. Furthermore, while the lesioned and the control rats produced similar levels of CRs when the target stimulus was presented by itself, the lesioned rats responded more than did the controls to the stimulus compound. Multiple ANOVAs using surgery (Hip vs. Con) as a between-subject factor and trial type (noise vs. light-noise) and trials ( 1 or 2 ) as withinsubjects factors revealed a significant main effect of trial type $[F(1,14)=39.94, p<.01]$ and a significant trial type $\times$ trial interaction $[F(1,14)=8.1, p<.05]$, but no other main effect or interaction was significant (largest $F=$ 4.31 , for surgery $\times$ trial type interaction).

Even though the surgery $x$ trial type interaction failed (narrowly) to reach significance $[F(1,14)=4.31, p=$ $.055]$, the pattern of differences found are consistent with the hypothesis that the rats with hippocampal lesions were impaired in learning inhibition. It would seem imprudent to ignore this possibility given its importance relative to the purpose of this study and given that prior studies (e.g., Solomon, 1977) have not examined the effects of hippocampal lesions on summation test performance. The observation that the lesioned rats responded more than the controls to the stimulus compound is exactly what we would expect if the hippocampal lesioned rats were impaired relative to the controls in learning inhibition.

Extinction test. Figure 4 shows mean responding by the lesioned and the control rats during the extinction of the noise stimulus. The figure shows that the lesioned and the control rats did not differ in their conditioned responses in the beginning of the extinction phase. After three extinction sessions, the control rats substantially reduced the amount of conditioned responses, whereas the lesioned rats still maintained a high level of conditioned responses. This observation was confirmed with a multiple ANOVA using surgery (Hip vs. Con) as a between-subjects factor and block (1 or 2), trials (1-12), and period (pre-CS vs. CS) as within-subjects factors. The statistical analyses showed a significant main effect of period $[F(1,14)=42.4$, $p<.01]$ and significant surgery $\times$ block $[F(1,14)=8.7$, $p<.05]$ and surgery $\times$ block $\times \operatorname{trial}[F(11,154)=1.9, p<$ $.05]$ interactions. There was no other significant main effect or interaction involving either surgery or period (largest $F=1.67$, for the surgery $\times$ trial interaction). To make sure that the surgery $\times$ block interaction was not due to differences in responding during the pre-CS period alone, we further analyzed the data from the CS period by itself. A multiple ANOVA using surgery (Hip vs. Con) as a between-subjects factor and block ( 1 or 2$)$ and trials (1-12) as within-subjects factors yielded a significant main effect of block $[F(1,14)=14.4, p<.01]$ and a significant surgery $\times$ block interaction $[F(1,14)=5.7, p<$ $.05]$, thus substantiating the claim that hippocampallesioned rats were impaired in extinguishing CR to the CS.

Retraining and retardation test. After the extinction phase, all the subjects were given retraining with the orig-

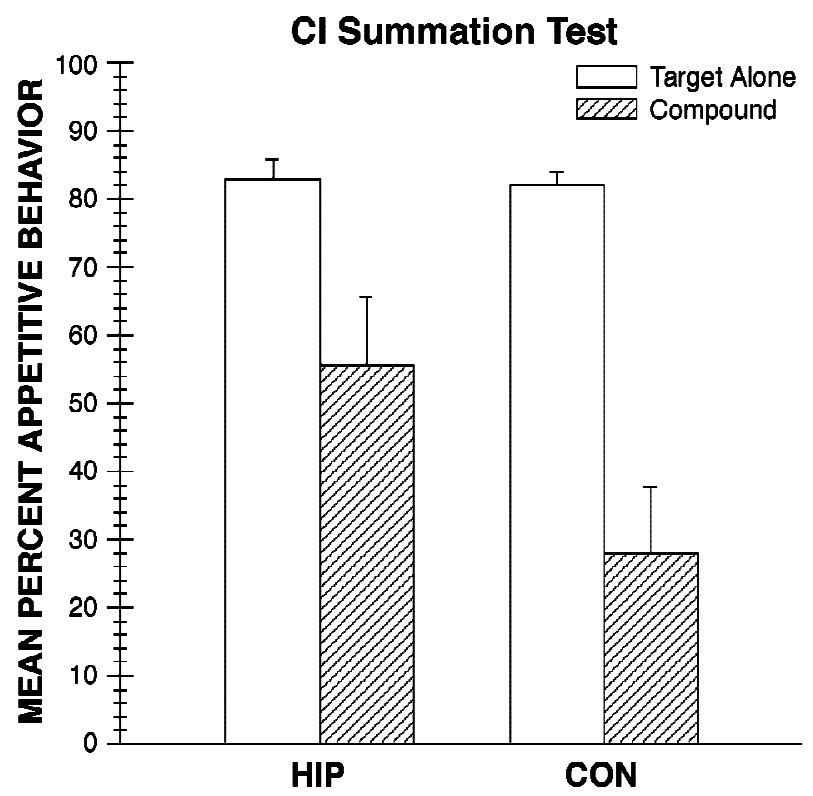

Figure 3. Appetitive behavior by hippocampal-lesioned (HIP) and control $(\mathrm{CON})$ rats during the summation test (i.e., $\mathrm{N}-$ vs. $\mathrm{LN}-$ ) after conditioned inhibition (CI) training. The bars on the left show conditioned response (CRs) by lesioned rats, and the bars on the right show $C R s$ by controls. The open bars represent appetitive behavior measured when the feature stimulus was presented alone, and the hatched bars represent appetitive behavior measured when the feature stimulus was presented in compound with the target stimulus. 


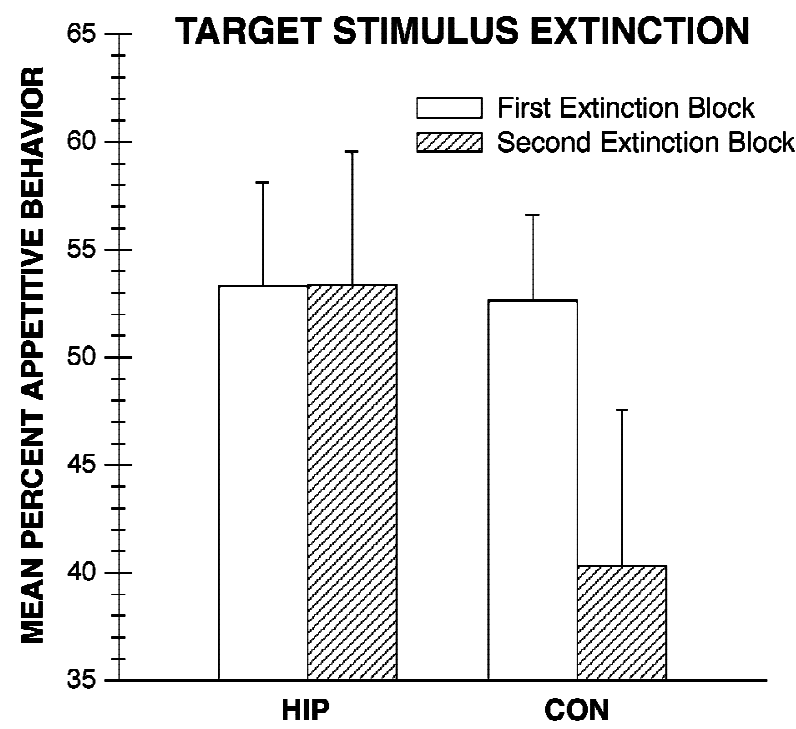

Figure 4. Appetitive behavior by the hippocampal-lesioned (HIP) and the control (CON) rats during the extinction phase (i.e., $\mathbf{N}-$ ). The bars on the left show conditioned responses (CRs) by the lesioned rats, and the bars on the right show CRs by the controls. The open bars represent appetitive behavior during the first 12 trials, and the hatched bars represent appetitive behavior during the next 12 trials.

inal CI procedure. Both the control and the lesioned rats quickly reacquired the discrimination, and the two groups did not differ during the retraining phase. The mean percentages of appetitive behavior for groups Hip-T + , HIPLT - , CON-T+, and CON-LT - were $84.12 \%, 40.27 \%$, $79.43 \%$, and $41.01 \%$, respectively. A multiple ANOVA using surgery (Hip vs. Con) as a between-subjects factor and trial type (tone vs. light-tone), session (1-4), and trial block (1 or 2) as within-subjects factors yielded a significant main effect of trial type $[F(1,14)=118.5, p<.01]$. None of the other main effects or interactions reached significance (largest $F=3.12$, for the main effect of trial).

Figure 5 shows mean conditioned responses by the lesioned and the control rats during the retardation test phase. Over eight sessions of light followed by US presentations, both the lesioned and the control rats learned to make conditionedresponses to the light. The two groups did not differ systematically during the retardation test. A multiple ANOVA using surgery (Hip vs. Con) as a between-subjects factor and session (1-8) and trials (1-8) as within-subjects factors yielded significant main effects of session $[F(7,98)=19.0, p<.01]$ and trial $[F(7,98)=$ $3.7, p<.01]$. There was no other significant main effect or interaction (largest $F=1.0$, for the surgery $\times$ session $\times$ trial interaction).

\section{DISCUSSION}

As was noted earlier, Solomon (1977) reported that lesion of the dorsal hippocampus failed to prevent the acquisition of Pavlovian $\mathrm{CI}(\mathrm{A}+, \mathrm{XA}-)$ of the nictitating membrane response in rabbits. In Solomon's study, aspiration lesions aimed at the dorsal hippocampi left open the possibility that the extent of hippocampal damage in his experiment was not large enough for impairments in inhibitory learning to be expressed. The present experiment provided a more specific and more complete ablation of the dorsal and ventral hippocampus in the rat to assess its role in solving the CI task. Second, we also included additional tests to assess the possibility that impaired inhibitory learning on the part of hippocampal-lesioned rats would be revealed under conditions that were different from those examined by Solomon.

The results from the acquisition phase of the present experiment replicated Solomon's (1977) finding that hippocampal-lesioned animals were not impaired in learning to inhibit responding to the nonreinforeced compound in the classic CI paradigm. In an attempt to clarify whether hippocampal lesioned and control rats solved the problem by using different strategies or different levels of inhibitory learning, we also conducted a posttraining retardation test at the end of the experiment. The result of this test also confirmed Solomon's findings of no significant difference between hippocampal-lesioned and control subjects. These results support Solomon's conclusion that the hippocampus is not essential for solving the CI task.

In addition to replicating the training and testing phases (i.e., CI acquisition and retardation test) reported by Solomon (1977), we also conducted some additional tests to further investigate the role of the hippocampus in in-

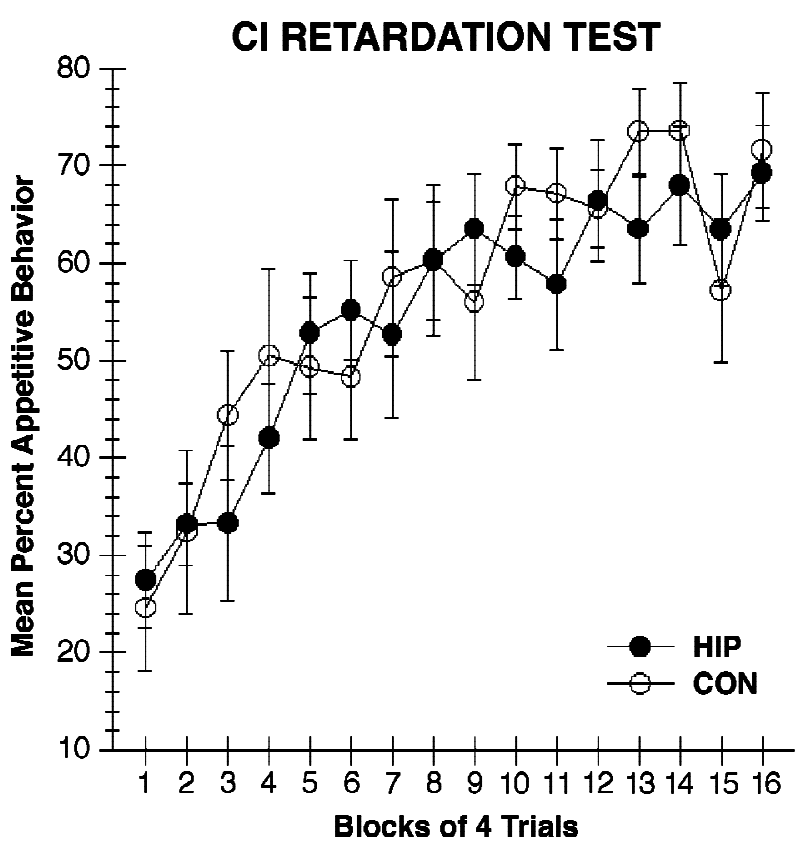

Figure 5. Acquisition of appetitive behavior by hippocampallesioned (HIP) and control (CON) rats in the retardation test $(\mathrm{L}+)$. The closed circles represent appetitive behavior by the lesioned rats, and the open circles represent appetitive behavior by the controls. 
hibitory learning. According to Rescorla (1969), both the retardation test and the summation test are necessary to assess the nature of $\mathrm{CI}$, the reason being that only when both tests are used can the experimenter rule out both generalization decrement and latent inhibition as alternative explanations for the pattern of results (see Rescorla, 1969). Solomon did not report any results from a summation test. In the present experiment, when the inhibitor (light) was paired with another excitatory stimulus (noise), both the lesioned and the control subjects suppressed responding to the novel light-noise compound, suggesting that the subjects had acquired an inhibitory association to the light and that inhibitory associative strength transferred to a different CS in the summation test.

Even though a negative summation effect is consistent with our idea that the hippocampus is not involved in the learning of direct inhibitory association from the CI task, a complete and circumspect interpretation of the findings cannot simply ignore the fact that the controls appeared to show more transfer of inhibition than did the hippocampallesioned rats (marginally significant, $p=.055$ ) during the summation test. The difference is consistent with the possibility that the hippocampal-lesioned rats acquired less inhibition during original training than did the controls. Thus, what needs to be explained is how hippocampal lesions could impair performance in the summation test, but not during acquisition of CI.

One important difference between the two procedures is that $\mathrm{A}+$ and $\mathrm{XA}-$ trials are intermixed throughout $\mathrm{CI}$ acquisition, whereas compound presentations of the inhibitor and the transfer target (XB-) are given in the summation test only after target training. Separate, prior training of the transfer CS used in summation testing might result in the formation of a stronger excitatory association between that cue and the US, as compared with the CS used in standard CI training. Accordingly, one possible explanation is that the transfer excitor had more excitation than did the original stimulus, making it harder to inhibit responding to it. Another possible explanation is based on the common assumption that an inhibitory CS-US association develops to the extent that the US memory is activated on trials when the actual US does not occur. In other words, with respect to the treatment of the transfer CS, the summation procedure is quite like extinction, in that both involve the nonreinforcement of a highly excitatory CS. It is possible that the marginally significant finding obtained in the present summation test indicates weaker acquisition of inhibition to the transfer CS as a consequence of extinction, instead of (or in addition to) weaker stimulus control by the previously established conditioned inhibitor. Although admittedly speculative, both interpretations are consistent with the view that hippocampal-lesioned rats are impaired in forming inhibitory associations to cues that are already embedded in an excitatory association.

As was noted previously, extinction appears to involve the formation of an inhibitory association to a CS that is already embedded in a concurrent excitatory association with the same US. The results of the extinction test that occurred following summation testing revealed that the same subjects as those that were not impaired in solving the CI task were impaired in learning to extinguish conditioned responding to a stimulus that had previously been paired with the presentation of the US. This pattern of result is consistent with the hypothesis that the hippocampus may be involved with the formation of inhibitory associations involving cues that are already embedded in an excitatory association with the US (Chan et al., 2001). This outcome indicates (1) that the extinction and CI procedures yield different hippocampal-dependent and hippocampalindependent types of inhibitory learning or (2) that both procedures produce the same type of inhibitory learning but involve parameters (e.g., rate of conditioning, asymptotic strength of conditioning, etc.) that are sensitive to the effects of hippocampal lesions.

There is also a third procedure that appears to produce inhibitory learning that is, in some ways, distinct from that produced by extinction and by Solomon's (1977) CI procedure. Holland $(1989,1992)$ reported that if the $\mathrm{A}+$, $\mathrm{AX}$ - problem used by Solomon is modified so that, on compound trials, the $\mathrm{X}$ and the A stimuli are presented serially $\left(\mathrm{X} \rightarrow \mathrm{A}^{-}\right)$instead of simultaneously $(\mathrm{XA}-), \mathrm{X}$ does not enter into a direct association with the US. Rather, serial training endows $\mathrm{X}$ with the capacity to modulate the effectiveness of the association between Stimulus A and the US. Recently, Holland et al. (1999) found that rats with neurotoxic lesions of the hippocampus were significantly impaired, relative to controls, in learning the serial $\mathrm{A}+, \mathrm{X} \rightarrow \mathrm{A}-$ problem. These researchers proposed that hippocampal damage interfered with the development of $X$ 's modulatory function. However, other evidence has indicated that such modulation will not occur unless Stimulus A has been exposed to both excitatory and inhibitory training (e.g., Bouton, 1993; Swartzentruber \& Rescorla, 1994). Consistent with the present analysis, if the serial $\mathrm{A}+, \mathrm{XA}-$ training embeds Stimulus A in concurrent excitatory and inhibitory associations and if the hippocampus is needed to form inhibitory associations involving stimuli that are also embedded in excitatory associations, then removing the hippocampus should disrupt performance on the serial $\mathrm{A}+, \mathrm{X} \rightarrow \mathrm{A}-$ problem. Moreover, on the basis of this analysis, impaired performance would be expected to take the form of elevated responding on nonrewarded $\mathrm{X} \rightarrow \mathrm{A}-$ compound trials. This was precisely the pattern of results reported by Holland et al. (1999). This interpretation should be viewed, at best, as an interesting hypothesis until (1) direct evidence is obtained that $\mathrm{A}+$ $\mathrm{X} \rightarrow \mathrm{A}-$ training results in the formation of concurrent excitatory and inhibitory associations and (2) the generality of the effects of hippocampal lesions on performance in serial $\mathrm{A}+, \mathrm{X} \rightarrow \mathrm{A}-$ problems is established.

It also makes sense to consider the generality of the present findings. In the attempt to replicate Solomon's (1977) original experiment, we used the same stimuli for the present experiment. As a result, the different stimuli used for the CI phase and the extinction phase were not counterbalanced, leaving open the possibility that le- 
sioned animals are impaired in learning inhibition only to the noise, but not to the tone (or other stimuli). We think this possibility is unlikely. We reported previously rats with selective ibotenate lesion of the hippocampus are impaired in learning extinction even when the stimulus is a tone identical to the one used in the present experiment (Benoit et al., 1999). Furthermore, numerous studies employing diverse stimuli, response measures, and training conditions have reported impaired extinction following hippocampal damage (see Chan et al., 2001). The results of recent studies have also provided autoradiological evidence that seems consistent with the results that are reported here. For example, Nair and Gonzalez-Lima (1999) reported that rat pups show significantly slower rates of extinction in a runway task at Postnatal Day 12 than at Postnatal Day 17. Using brain metabolic mapping techniques, these researchers found that improvement in extinction performance between Postnatal Days 12 and 17 was positively correlated with increased metabolic activity in the CA1 and CA 3 cell fields of the hippocampus. Increased activity in these hippocampal cell fields was not reported in an earlier study that used a similar metabolic analysis of the brain activity of adult rats during standard Pavlovian CI training (e.g., McIntosh \& Gonzalez-Lima, 1995).

It is also important to point out that hippocampal lesions do not make it impossible for rats to learn to extinguish responding to a cue. What we have observed is that hippocampal-lesioned rats are slower than intact rats in learning extinction to an appetitive stimulus. As a result, in studies in which terminal performance or large trial blocks during the extinction phase were looked at, any difference in inhibitory learning might not have been noticed. Subjects with previous experience that provided opportunity to form inhibitory associations between the same CS and the US also would not be expected to show impairment in extinction or inhibitory learning. These factors need to be taken into consideration in order to evaluate earlier reports of hippocampal lesion effects in learning extinction.

Learning theorists suggest that extinction and Pavlovian CI training involve the formation of different associative structures (for a review, see Chan et al., 2001). If inhibitory associations develop more rapidly within one type of associative structure than in another and if hippocampal damage reduces somewhat the rate at which inhibitory association formation takes place, this effect might be detected more easily with an extinction procedure than with CI training. Thus, to understand more about the nature of the performance deficit produced by removing the hippocampus, it will be necessary to assess the effects of hippocampal damage not only on learning problems that involve different types of associative structure, but also on different rates and strengths of inhibitory association formation.

The results presented in the present report are consistent with the idea that the hippocampus is involved in the formation of inhibitory associations to cues that are already embedded in a concurrent excitatory association with the same US. Another possible interpretation of this result is Hirsh's (1974) contextual label hypothesis that the hippocampus is involved in the discrimination between two associations with the same CS. In this case, the two associations are a CS-US association and a CS-no-US association. This interpretation is reminiscent of Konorski's (1967) idea that Pavlovian inhibitory associations are essentially CS-no-US associations. This conceptual framework is very similar to our idea that the hippocampus is involved in learning inhibition to a CS that is already embedded in a concurrent excitatory association (see Chan et al., 2001). One difference is that we make a more specific prediction that the hippocampus is involved only in learning the inhibitory (i.e., CS-no-US), but not the excitatory (i.e., CS-US), association. This hypothesis also seems to encompass a number of the most robust appetitive Pavlovian conditioning behavioral effects of hippocampal damage. These include the findings from general behavioral activity, context conditioning, simple extinction, trial spacing effects, certain types of complex discrimination learning problems, and even feeding behavior (see Chan et al., 2001 for a review). It should also be pointed out that impairments exhibited by hippocampal animals in spatial or declarative memory tasks may be based, at least in part, on deficits in inhibitory learning like those in extinction (see Chan et al., 2001). However, the nature of inhibitory learning in spatial and declarative tasks must be better specified before this possibility can be evaluated experimentally.

\section{REFERENCES}

Barnet, R. C., \& Miller R. R. (1996). Temporal encoding as a determinant of inhibitory control. Learning \& Motivation, 27, 73-91.

Benoit, S. C., Davidson, T. L., Chan, K.-H., Trigilio, T., \& JARRARD, L. E. (1999). Pavlovian conditioning and extinction of context cues and punctate CSs in rats with ibotenate lesions of the hippocampus. Psychobiology, 27, 26-39.

Bouton, M. E. (1993). Context, time, and memory retrieval in the interference paradigms of Pavlovian learning. Psychological Bulletin, 114, 80-99.

Bouton, M. E. (1994). Conditioning, remembering, and forgetting. Journal of Experimental Psychology: Animal Behavior Processes, 20, 219-231.

Bouton M. E., \& Nelson, J. B. (1998). Mechanisms of feature-positive and feature-negative discrimination learning in an appetitive conditioning paradigm. In N. A. Schmajuk \& P. C. Holland (Eds.), Occasion setting: Associative learning and cognition in animals (pp. 69112). Washington, DC: American Psychological Association.

Chan, K.-H., Morell, J. R., Jarrard, L. E., \& Davidson, T. L. (2001). Reconsideration of the role of the hippocampus in learned inhibition. Behavioural Brain Research, 119, 111-130.

Delamater, A. R. (1996). Effects of several extinction treatments upon the integrity of Pavlovian stimulus-outcome associations. Animal Learning \& Behavior, 24, 437-449.

HIRSH, R. (1974). The hipppocampus and contextual retrieval of information from memory: A theory. Behavioral Biology, 12, 421-444.

Holland, P. C. (1985). The nature of CI in serial and simultaneous feature negative discriminations. In R. R. Miller \& N.E. Spear (Eds.), Information processing in animals: Conditioned inhibition (pp. 267297). Hillsdale, NJ: Erlbaum.

Holland, P. C. (1989). Occasion setting with simultaneous compounds in rats. Journal of Experimental Psychology: Animal Behavior Processes, 15, 183-193.

Holland, P. C. (1992). Occasion setting in Pavlovian conditioning. In 
D. L. Medin (Ed.), The psychology of learning and motivation (Vol. 28, pp. 69-125). San Diego: Academic Press.

Holland, P. C., Lamoureux, J. A., Han, J.-S., \& Gallagher, M. (1999). Hippocampal lesions interfere with Pavlovian negative occasion setting. Hippocampus, 9, 143-157.

JARRARD, L. E. (1989). On the use of ibotenate acid to lesion selectively different components of the hippocampal formation. Journal of Neuroscience Methods, 29, 251-259.

KonORSKI, J. (1948). The physiological bases of memory. Mysl Wspolczesnosc, 5, 214-232.

KoNORSKI, J. (1967). Integrative activity of the brain. Chicago: University of Chicago Press.

Matzel, L. D., Gladstein, L., \& Miller, R. R. (1988). Conditioned excitation and conditioned inhibition are not mutually exclusive. Learning \& Motivation, 19, 99-121.

McIntosh, A. R., \& Gonzalez-Lima, F. (1995). Functional network interactions between parallel auditory pathways during Pavlovian conditioned inhibition. Brain Research, 683, 228-241.

NaIr, H. P., \& GonZalez-Lima, F. (1999). Extinction of behavior in infant rats: Development of functional coupling between septal, hippocampal, and ventral tegmental regions. Journal of Neuroscience. 19, 8646-8655.

PAXINos, G., \& WATSON, C. (1998). The rat brain in stereotaxic coordinates. San Diego: Academic Press.
Pearce, J. M. (1987). A model for stimulus generalization in Pavlovian conditioning. Psychological Review, 94, 61-73.

RESCORLA, R. A. (1969). Conditioned inhibition of fear resulting from negative CS-US contingencies. Journal of Comparative \& Physiological Psychology, 67, 504-509.

Solomon, P. R. (1977). Role of the hippocampus in blocking and conditioned inhibition of the rabbit's nictitating membrane response. Journal of Comparative \& Physiological Psychology, 91, 407-417.

Swartzentruber, D., \& Rescorla, R. A. (1994). Modulation of trained and extinguished stimuli by facilitators and inhibitors. Animal Learning \& Behavior, 22, 309-316.

WAGNER, A. R. (1981). SOP: A model of automatic memory processing in animal behavior. In N. E. Spear \& R. R. Miller (Eds.), Information processing in animals: Memory mechanisms (pp. 5-47). Hillsdale, NJ: Erlbaum.

Williams, D. A., \& Overmier, J. B. (1988). Some types of conditioned inhibitors carry collateral excitatory associations. Learning \& Motivation, 19, 345-368.

Williams, D. A., Overmier, J. B., \& LoLordo, V. M. (1992). A reevaluation of Rescorla's early dictums about Pavlovian conditioned inhibition. Psychological Bulletin, 111, 275-290.

(Manuscript received October 22, 2002; revision accepted for publication February 10, 2003.) 